\title{
MATHEMATICAL MODELS FOR FLOOD HAZARD ASSESSMENT
}

\author{
D. DE WRACHIEN ${ }^{1} \&$ S. MAMBRETTI ${ }^{2}$ \\ ${ }^{1}$ Department of Agricultural Engineering, State University of Milano, Italy. \\ ${ }^{2}$ DIIAR, Politecnico di Milano, Italy.
}

\begin{abstract}
One-third of the annual natural disasters and economic losses, and more than half of the respective victims are flood-related. Knowledge and advanced scientific tools play a role of paramount importance in the strain of coping with flooding problems. The paper, after a brief discussion of the terms hazard, vulnerability, and risk, presents the governing equations for 1D, 2D, and 3D models, together with examples of their applications and discussion of the carried out results. It is shown that $1 \mathrm{D}$ models can be used only in very particular cases, but actually they are still the most reliable and robust of all the models; wide spread use of 2D models is justified by the relatively easiness in their use, and because the time consumption is still quite reduced. As for 3D models, their use in environmental hydraulic is still limited to very peculiar cases, because of the difficulties encountered in their use and the impressive time consumption they require, which in the largest cases necessitates the dramatic simplification of the topography or of the turbulence models that implies poor quality results.

Keywords: case studies, flood hazard, mathematical models.
\end{abstract}

\section{INTRODUCTION}

One-third of the annual natural disasters and economic losses, and more than half of the respective victims are flood-related. These hazards are likely to become more frequent and more relevant in the future, due to the effects of increase in population, urbanization, land subsidence, and the impacts of climate change.

Knowledge and advanced scientific tools play a role of paramount importance in the strain of coping with flooding problems. In this context, flood modeling represents the basis for effective flood mitigation.

The modeling approach aims to provide the best means for assessing and, subsequently, reducing the vulnerability of rural and urban flood prone areas.

By using models, an attempt is made to replace trial and error based strategies, as practised in the past, with more physically based measures of flood management and control. Mathematical models are the best tools, nowadays available, for the design of efficient flood protection strategies and excellent supporters of decision-makers.

With reference to these issues, the paper provides a review and a general description of the main features of the models currently used in flood management, which range from 1D to 3D. Moreover, to highlight the effectiveness and the resilience of these tools, some case studies of flood mitigation and hazard assessment are presented.

\section{RISK, HAZARD, VULNERABILITY}

Before the description of a possible methodology to carry out a risk analysis and its application to a number of case studies, it is necessary to provide some preliminary definitions, because of a certain lack of uniformity in the use of terms.

In the report of the International Commission on Irrigation and Drainage (ICID) [1] hazard, $H$ is defined as the 'probability of occurrence of a potentially dangerous event in a fixed time 
range and in a fixed area'. In this definition the concepts of time and space are explicitly stated, but the event magnitude concept is not mentioned. The Einstein [2] approach is quite different. The hazard concept definition is based on the geometrical and mechanical characteristics of the natural phenomenon. In this way the concepts of magnitude and area of potentially dangerous events are explicit and hazard is defined as the 'probability of occurrence of a danger in a fixed time range'.

In practice, hazard $H$ is described in different ways in relation with the topic/issue (earthquakes, landslides, debris flows, etc.) dealt with. The return period is often used to characterize the events with fixed magnitude in a specific area. To this regard, a relevant aspect, neglected in Varnes' definition, is the spatial propagation of the phenomenon. If the propagation is neglected, the risk analysis results are incomplete, because it is limited to the beginning of the process. It is equally important, instead, the probability that the wave reaches at a certain time, a certain place. In this case it seems more appropriate to define it as induced hazard.

Exposition $E$ can be defined as the 'probability that a certain element be exposed to the risk when an event of fixed magnitude, in a fixed time range and in a fixed area, occurs'. Different authors define $E$ as the 'probability that an element be affected by a fixed hazard'. Sometimes exposition is also defined as a 'quantitative index to sum up the number of persons and goods potentially subject to the event'.

Vulnerability $V$ can be defined as the inverse of the resilience, where resilience describes the capacity of ecosystems to react against the stress. Thus, vulnerability represents the territorial system tendency to suffer damages during an extreme event. With reference to people, vulnerability can be assessed as the characteristics and situation of a person or group that influence their capacity to cope with, resist, and recover from the impact of a natural hazard.

Risk $R$ is the total damage caused by a specific event, and it can be obtained as a function of hazard, exposition, and vulnerability:

$$
R=H \cdot V \cdot E
$$

In [3] detailed discussions about this expression and the different terms involved are reported. However, in this paper only the evaluation of the hazard $H$ will be considered, trying to frame a procedure for the assessment of a quantitative value.

In case of flood, hazard is given by the characteristics of the flood wave, which can be described by the depth $h$ and the velocity $V$. The combination of these terms can produce different results. A quite spread method is the determination of a critical value of the momentum $M$ on a structure, which can be written as:

$$
M=\frac{1}{2} \cdot \gamma \cdot h^{2} \cdot L+\rho \cdot h \cdot L \cdot V^{2}
$$

where $L$ is the length of the structure and $\gamma, \rho$ are the specific weight and the density of the fluid (water, in this case). To compute the specific momentum $S$, the above value has to be divided by the area $h \cdot L$; introducing into the eqn (2) the numerical values: $\gamma=9806 \mathrm{~N} / \mathrm{m}^{3}$ and $\rho=1000 \mathrm{~kg} / \mathrm{m}^{3}$, the velocity can be expressed as:

$$
V=\sqrt{\frac{S-\frac{1}{2} 9806 \cdot h}{1000}}
$$

In Fig. 1 the eqn (3) has been plotted for three different specific momentum $S$, obtaining the determination of different hazard $H$ conditions. Increasing the specific momentum of the 


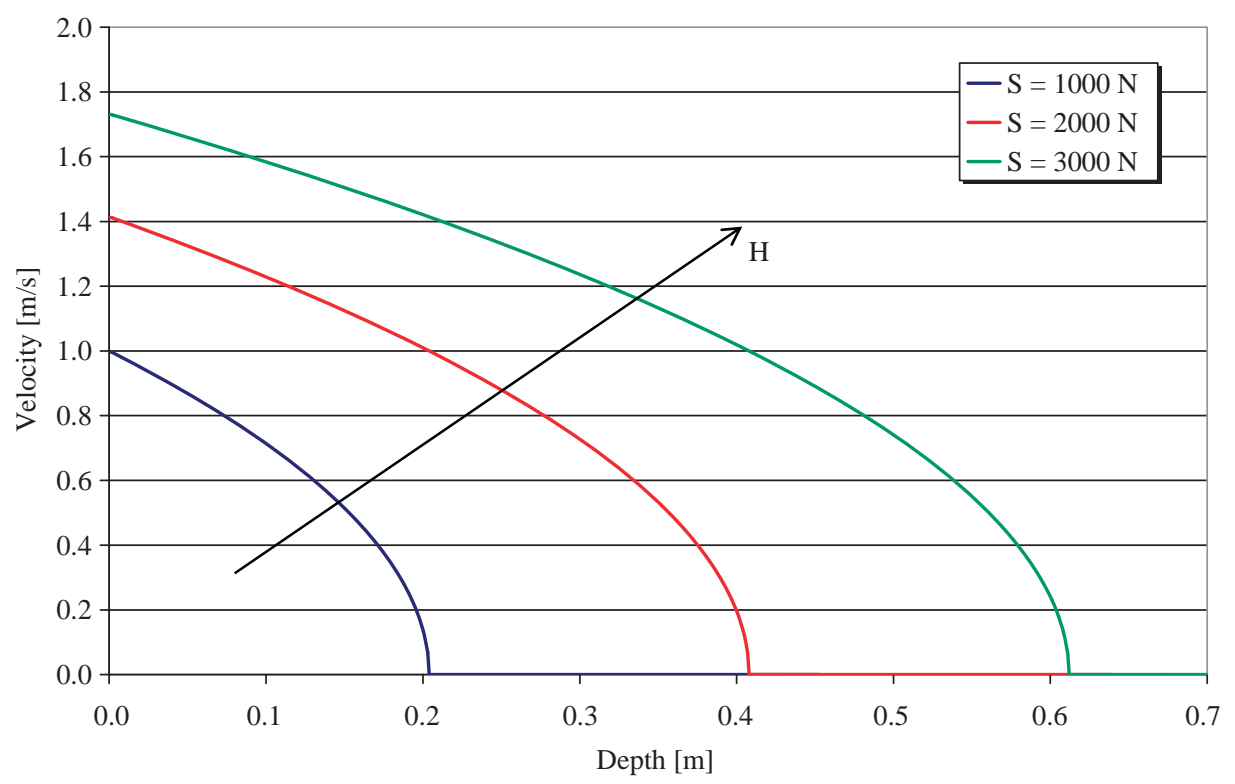

Figure 1: Determination of hazard level.

flow obviously increases the hazard. It is therefore possible to assess the hazard level once the flow characteristics are known.

Mathematical models aim to compute the flow characteristics, namely the depth $h$ and the velocity $V$ to allow the determination of the hazard in the flooded area.

In Italy, the River Authorities, which are in charge for the management of the river works and for planning the activities in the areas related to rivers, divide the flood prone areas in three subareas, which are:

- 'A' the area flooded by a discharge equal to $80 \%$ of a flood of 200 -year return period;

- 'B' the area flooded by a discharge of a flood of 200-year return period;

- ' $\mathrm{C}$ ' the area flooded by a discharge of a flood of 500-year return period, or a 'catastrophic' event if available.

The definition of these areas is assumed by the Authorities for the most important rivers. Obviously, 'A' and 'B' areas are parts of the river itself and no activities are allowed. On the other hand, ' $C$ ' areas can be very large, and it is not possible to forbid any activities there. To this end, the Authorities define other subareas with different degrees of potential hazard where different activities are possible. For instance, the Lombardy Region defines four subareas within ' $\mathrm{C}$ ' areas, which are:

- 'R1' area, where slight risk is expected, and therefore where no specific constrains are determined for the urbanization;

- 'R2' area, where medium risk has been assessed and where, should further urbanization be scheduled, appropriate countermeasures have to be taken for risk reduction and where the municipality may require specific studies about hydro-geological features;

- 'R3' area, where high risk has been assessed and therefore no further urbanization should be allowed, but for public use, while restoration is allowed and the application of 
countermeasures against flood are recommended; moreover documents concerning the hydro-geological conditions must be produced;

- 'R4' area, very high risk, no urbanization is allowed and restoration is allowed only if vulnerability reduction is achieved; strictly forbidden are all the chemical and petrol-chemical activities along with garbage dumps.

\section{1D MATHEMATICAL MODELS}

\subsection{Governing equations, applications, and limits}

The 1D approach to flood routing is based on the continuity and momentum equations for unsteady flow of an incompressible fluid, expressed in a conservative form [4].

This set of equations, known as the De Saint Venant (SV) equations, can be written in compact form as:

$$
\frac{\partial U}{\partial t}+\frac{\partial E}{\partial x}=S
$$

where

$$
U=\left(\begin{array}{c}
h \\
u h
\end{array}\right) \quad E=\left(\begin{array}{c}
u h \\
u^{2} h+\frac{1}{2} g h^{2}
\end{array}\right) \quad S=\left(\begin{array}{c}
0 \\
g h\left(S_{0_{x}}-S_{f}\right)
\end{array}\right)
$$

and $t$ is the temporal coordinate; $h$ the water depth; $u$ the water velocity; $g$ the acceleration due to gravity; $S_{0}$ the bed slope; and $S_{f}$ the bed resistance term (or friction slope).

As obvious, in this case the direction of the flow has to be known in advance, which makes the estimation of a flooded area very difficult, which by definition holds a $2 \mathrm{D}$ behavior. In the past, when the 2D models were not as wide spread as today, practitioners used these equations to model the flood prone areas characterized by a network of 1D channels. This methodology, even if not very accurate and difficult to calibrate, is still used nowadays for preliminary evaluations and in particular cases.

Up-to-date models use both 1D and 2D equations to describe the flood: 1D to simulate the wave propagation inside the river, and $2 \mathrm{D}$ to simulate the wave in the flood-prone areas outside the river or the channel. In fact, while theoretically the simulation of 1D phenomena is possible with $2 \mathrm{D}$ models, the latter are not sufficiently accurate when applied to $1 \mathrm{D}$ cases.

Moreover, in few particular cases 1D models are efficient for the hazard assessment.

\subsection{A case study: hazard assessment for a gipsy camp in Milano}

In Milano, an authorized gipsy camp is located very close to the Lambro river. The camp was installed in the early $80 \mathrm{~s}$ and in that time no preliminary studies were carried out. It was found that the camp was inside the ' $\mathrm{B}$ ' area of the Lambro river, and therefore the only allowed activities to be performed were related to the vulnerability reduction. Figure 2 shows the area with the location of the camp. In the figure, different areas have been coloured: red areas that can be flooded with return period lower than 10 years, green areas expected to be flooded with 200-year return period, and yellow areas feasible to be flooded with 500-year return period. 


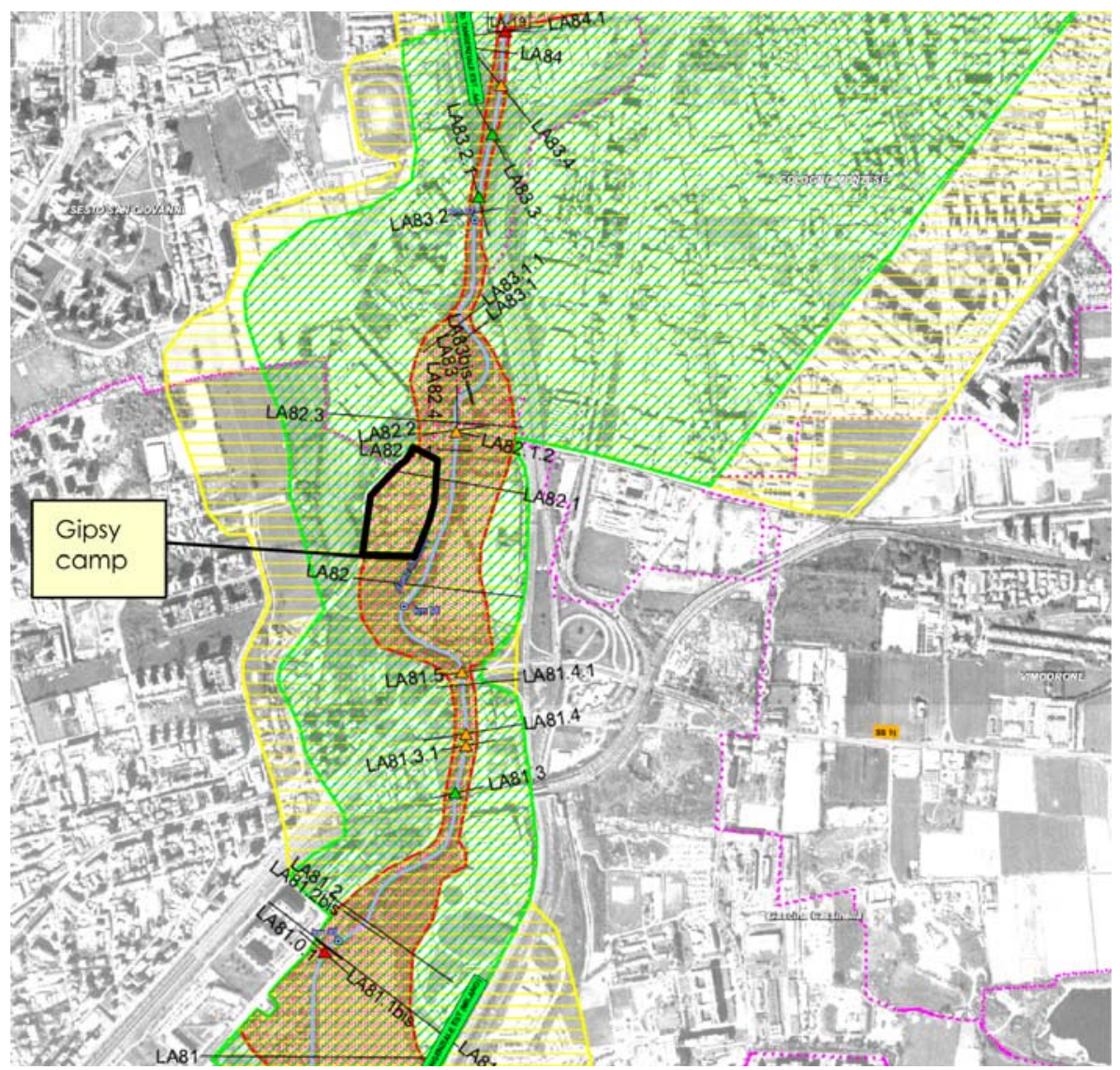

Figure 2: Lambro river: flood-prone areas.

To obtain a permanent and safe camp, some simulations have been carried out and countermeasures have been studied on the basis of the characteristics of the river and the location of the camp. To this end, the well-known HEC-RAS has been used to assess the maximum value of the discharge and to design the most suitable measures to evacuate the camp when a given threshold was exceeded.

\section{2D MATHEMATICAL MODELS}

\subsection{Governing equations, applications, and limits}

A good understanding of a complex flooding event can only be achieved by means of a 2D hydrodynamic approach based on the SV equations.

These equations can be written in compact form as:

$$
\frac{\partial U}{\partial t}+\frac{\partial E}{\partial x}+\frac{\partial F}{\partial y}=S
$$


where

$$
U=\left(\begin{array}{c}
h \\
u h \\
v h
\end{array}\right) \quad E=\left(\begin{array}{c}
u h \\
u^{2} h+\frac{1}{2} g h^{2} \\
u v h
\end{array}\right) \quad F=\left(\begin{array}{c}
v h \\
u v h \\
v^{2} h+\frac{1}{2} g h^{2}
\end{array}\right) \quad S=\left(\begin{array}{c}
0 \\
g h\left(S_{0_{x}}-S_{f x}\right) \\
g h\left(S_{0_{y}}-S_{f y}\right)
\end{array}\right)
$$

and $t$ is the temporal coordinate; $h$ the water depth; $u$ and $v$ the water velocities in the $x$ and $y$ axis respectively; $g$ the acceleration due to gravity; $S_{0}$ the bed slope; and $S_{f x}$ and $S_{f y}$ the bed resistance terms (or friction slopes) in the $x$ and $y$ axis respectively.

Equations (6) and (7) represent the 2D unsteady flow equations for an incompressible fluid expressed in a conservative form [4].

Numerical solutions are possible with finite differences, finite volumes, or finite elements. In the latter case (see e.g. [5]), the increased complexity of the numerical scheme is compensated by the extreme flexibility of the grid, usually assumed triangular, which allows a much better description of the flooded area than the usual rectangular grid.

In this case the flood can propagate in the two directions and the description of the wave is exhaustive.

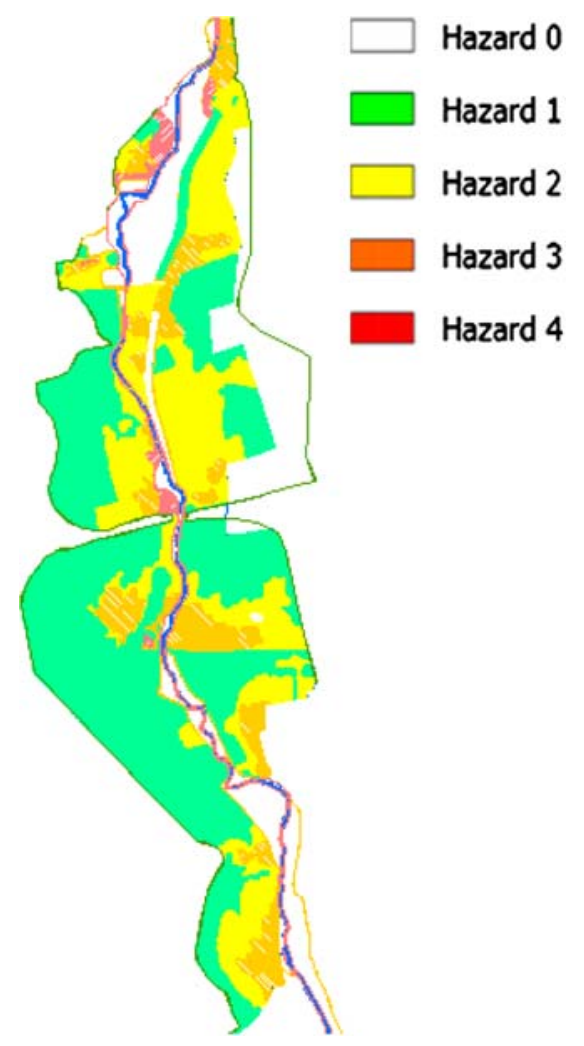

Figure 3: Hazard classes near the Lambro river in Milano. 


\subsection{The Lambro case study}

In Milano the flood-prone area of the Lambro river has been studied.

This case is different from that described in Section 3.2, because the area is much larger and the $1 \mathrm{D}$ simulation would not be significant. The part to be studied was the whole ' $\mathrm{C}$ ' area of the Lambro river within the Milano municipality.

A 2D model, based on the SV equations, has been built and calibrated using recording of a large flood that happened in 1951.

The model used for the simulations is an earlier version of the FLO-2D, with rectangular grid. The area, of about $20 \mathrm{~km}^{2}$, has been divided in squared cells of $50 \mathrm{~m} \times 50 \mathrm{~m}$, each characterized by the ground elevation and the Manning roughness coefficient. Simulations have been carried out with three different discharge values: one for the incipient flood, one for a flood of 200-year return period, and the last with a 500-year return period discharge.

The model gives the depth and velocity for each cell, function of the time. Maximum values of depth and velocity have been computed for each cell and to each cell a degree of hazard has been assigned, ranging from 1 (less dangerous) to 4 (extremely dangerous), according to the Lombardy Region requirements and using the procedure previously described.

Figure 3 shows the different hazard classes within the expected flooded area.

\section{3D MATHEMATICAL MODELS}

\subsection{Governing equations}

The 2D hydrodynamic models have to simulate the movement of a relatively shallow layer of water over a vast, nearly flat area. As soon as the depth of the water layer drops down to less than a given threshold (e.g. $0.5 \mathrm{~m}$ ), it becomes difficult to simulate the corresponding flow. The reason that this flooding phenomenon is unusually difficult to understand and predict in detail is its 3D behavior in an environment in which at any point the direction and the velocity of the wave will vary as a function of location $(x, y)$, ground level $(z)$, and time $(t)$. In these cases only 3D hydrodynamic models are feasible to properly describe the flood event.

3D models are based on the Navier-Stokes equations, i.e.:

$$
\left\{\begin{array}{l}
\rho \cdot \frac{D u}{D t}=-\frac{\partial p}{\partial x}+\frac{\partial \tau_{x x}}{\partial x}+\frac{\partial \tau_{y x}}{\partial y}+\frac{\partial \tau_{z x}}{\partial z}+\rho \cdot f_{x} \\
\rho \cdot \frac{D v}{D t}=-\frac{\partial p}{\partial y}+\frac{\partial \tau_{x y}}{\partial x}+\frac{\partial \tau_{y y}}{\partial y}+\frac{\partial \tau_{z y}}{\partial z}+\rho \cdot f_{y} \\
\rho \cdot \frac{D w}{D t}=-\frac{\partial p}{\partial z}+\frac{\partial \tau_{x z}}{\partial x}+\frac{\partial \tau_{y z}}{\partial y}+\frac{\partial \tau_{z z}}{\partial z}+\rho \cdot f_{z}
\end{array}\right.
$$

Where $u, v$, and $w$ are the velocity components in the direction $x, y$, and $z$ respectively, $p$ is the pressure, the parameters $\tau$ are those of the stress tensor, and $f$ is the friction.

As known, these equations are quite difficult to integrate but for very simple cases, usually related to simple 2D phenomena and in laminar flow; to actually use these equations, turbulence models are also required. 


\subsection{Applications, limits, and comments}

A 3D model has been used in the catchment of the Lambro river, to identify potentially flooded areas, assess the respective hazards, and to compare its effectiveness and resilience with respect to 2D hydrodynamic codes. The model is the well-known FLOW3D developed by the Flow Science Inc [6].

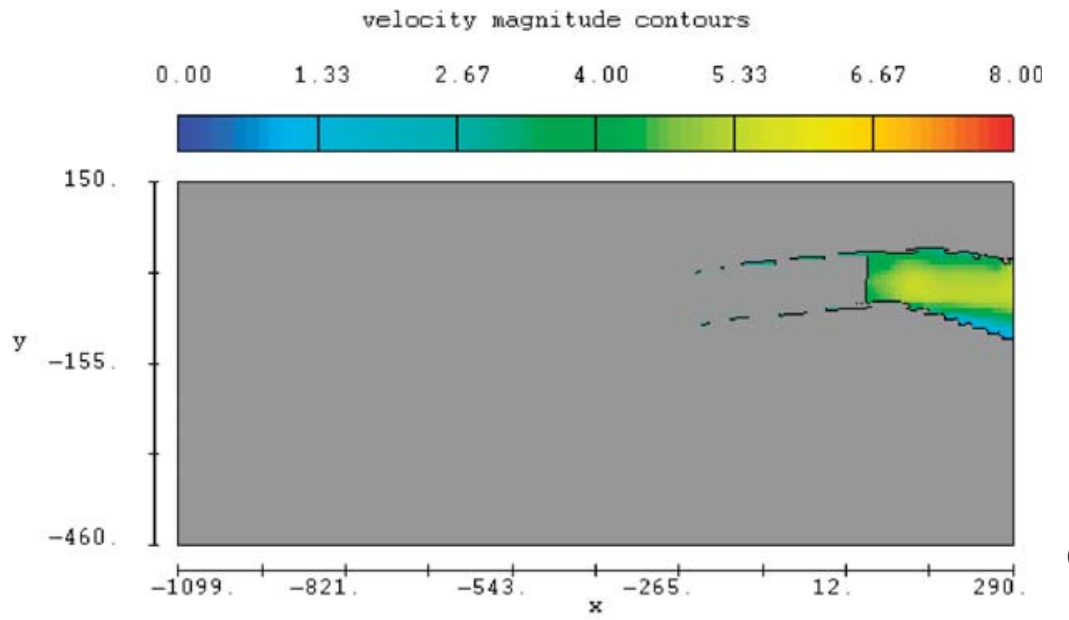

(a)

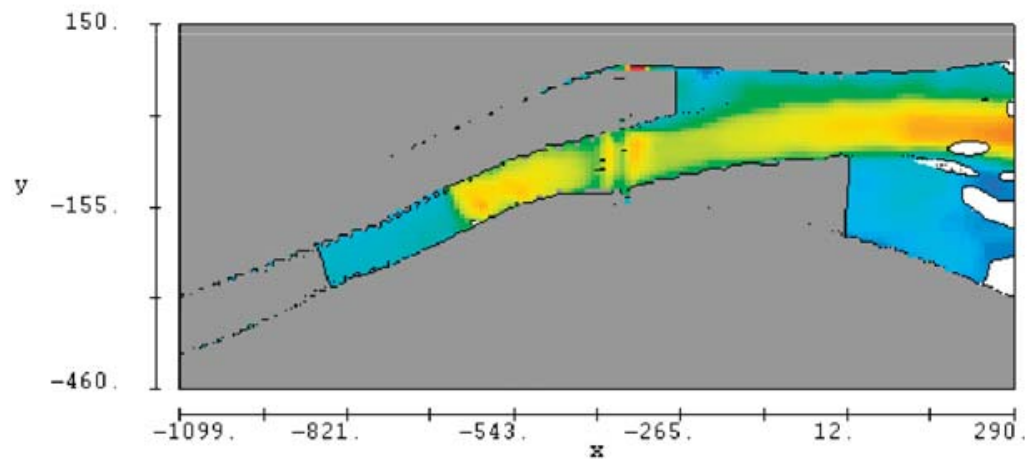

(b)

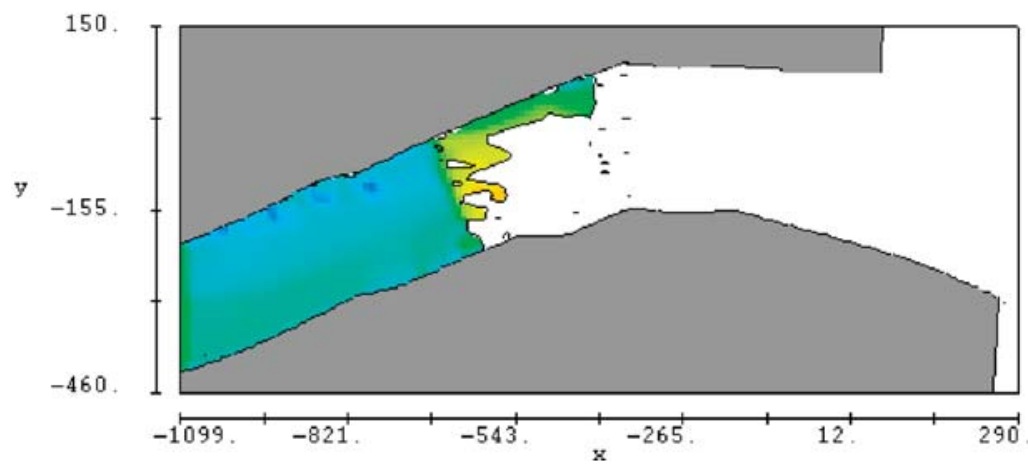

(c)

Figure 4: Velocities in the Vomano river at the outfall in the Adriatic Sea, at levels $-3.0 \mathrm{~m}$ asl (a), $+0.0 \mathrm{~m} \mathrm{asl}$ (b), $+3.0 \mathrm{~m} \mathrm{asl}$ (c) when the river discharge is equal to $1500 \mathrm{~m}^{3} / \mathrm{s}$. 
As a general comment, the 3D models are much more complex and the computational time is much more demanding for the reasons previously described. Moreover, a set of detailed data is indispensable to simulate a flood event. In fact, the flow wave moves through the plain in a course of few days or weeks and even a well-equipped authority is only able to make observations (water level, direction, and speed of the current) of such an event in only a few isolated spots.

A different and interesting application has been carried out by Da Deppo [7], who simulated the outfall of the Vomano river in the Adriatic Sea to compute the flow velocity and to check the stability of the embankments. In this case, although the simulated area is quite large, its dimensions allowed a complete description of the features of the flooded area taking into account that in the model the two existing bridges have been considered.

Figure 4 shows both the average and local (at different locations) velocities. The latter characteristic allows to assess its impact on structures like bridges and embankments.

On the whole, the use of 3D models should be limited to special cases, as [8]:

- when the results obtained by means of the 2D SV equations are not sufficiently reliable, due to the fact that the effects of the bottom friction and the exchange of the lateral momentum cannot be neglected;

- when the 3D flow pattern is relevant as on spreading of pollutants, floodplain risk assessment, and on setting up of emergency and evacuation plans.

\section{CONCLUSIONS}

Floods are among the most damaging of natural hazards, and are likely to become more frequent, more relevant, and more damaging in the future due to the effects of increase in population, urbanization, land subsidence and, to certain extent, the impact of climatic change.

A flood event is a natural event of great complexity. The hydrological parameters of a flood (magnitude, frequency, celerity, volume, and duration) reflect the stochastic behavior of precipitation, interception, infiltration, evapotraspiration, soil moisture, overland and ground flows, and river channel hydraulics.

Models not only help in understanding these flood phenomena, but are also essential for flood risk assessment of the current situation and for assessment of expected changes.

By using models an attempt is made to replace trial and error-based strategies as practised in the past with more physically based measures. The modeling tool aims to provide the best concept for assessing and reducing the vulnerability of rural and high-value urban floodprone areas as well as industrial zones.

Within this context, the paper provides a review and a general description of the main features and characteristics of the mathematical models currently used in flood management, mitigation, and control.

Mathematical models are useful tools for the design of efficient flood protection strategies and excellent supporters of decision makers, since they enable a complex evaluation of cost benefit analysis of particular proposal for flood protection measures. However, the choice of the best model and its use is a delicate matter that requires the experience and the skill of well-trained practitioners.

\section{REFERENCES}

[1] Duivendijk, J. van: Manual on Planning of Structural Approaches to Flood Management. ICID Guideline: New Delhi, India, 2005.

[2] Einstein, H.H., Special lecture: Landslide risk assessment procedure. Int. Symposium on Landslides. C. Bonnard (ed.) Landslide, 2, pp. 1075-1090, July 1988. 
[3] De Wrachien, D., Mambretti, S. \& Schultz, B., Flood Management and Risk Assessment in Flood-prone Areas: Measures and Solutions Accepted on Irrigation and Drainage, first published online: 23 jul 2010, DOI: 10.1002/ird.557.

[4] Lax, P.D. \& Wendroff, B., Systems of conservation laws Comm. Pure and Applied Mathematics 13, pp. 217-237, 1960. doi:http://dx.doi.org/10.1002/cpa.3160130205

[5] Garcia-Martinez, R., Gonzalez-Ramirez, N. \& O’Brien, J., Dam-break flood routing Chap. 4 in D. De Wrachien \& S. Mambretti (eds.) Dam-break. Problems, Solutions and Case Studies, WITPress: Southampton, 334 pp., October 2009, ISBN 978-1-84564-142-9.

[6] FLOW-3D User Manual - Ver. 9.4, Flow Science, Inc.: Santa Fe, New Mexico, 2009.

[7] Da Deppo, L., Opere per la salvaguardia del fiume Vomano, Relazione Idraulica, Regione Abruzzo, August 2004 (in Italian).

[8] Vreugdenhil, C.B., Numerical Methods for Shallow-Water Flow, Kulwer Academic Press: Dordrecht, Boston, London, 1998. 